\title{
Perivascular Cells in Diffuse Cutaneous Systemic Sclerosis Overexpress Activated ADAM12 and Are Involved in Myofibroblast Transdifferentiation and Development of Fibrosis
}

\author{
Paola Cipriani, Paola Di Benedetto, Piero Ruscitti, Vasiliki Liakouli, Onorina Berardicurti, \\ Francesco Carubbi, Francesco Ciccia, Giuliana Guggino, Francesca Zazzeroni, Edoardo Alesse, \\ Giovanni Triolo, and Roberto Giacomelli
}

\begin{abstract}
Objective. Microvascular damage is pivotal in the pathogenesis of systemic sclerosis (SSc), preceding fibrosis, and whose trigger is not still fully understood. Perivascular progenitor cells, with profibrotic activity and function, are identified by the expression of the isoform 12 of ADAM (ADAM12) and this molecule may be upregulated by transforming growth factor- $\beta$ (TGF- $\beta$ ). The goal of this work was to evaluate whether pericytes in the skin of patients with diffuse cutaneous SSc (dcSSc) expressed ADAM12, suggesting their potential contribution to the fibrotic process, and whether TGF- $\beta$ might modulate this molecule.

Methods. After ethical approval, mesenchymal stem cells (MSC) and fibroblasts (FB) were isolated from bone marrow and skin samples collected from 20 patients with dcSSc. ADAM12 expression was investigated in the skin and in isolated MSC and FB treated with TGF- $\beta$ by immunofluorescence, quantitative real-time PCR, and western blot. Further, we silenced ADAM12 expression in both dcSSc-MSC and -FB to confirm the TGF- $\beta$ modulation.

Results. Pericytes and FB of dcSSc skin showed an increased expression of ADAM12 when compared with healthy control skin. TGF- $\beta$ in vitro treatment induced a significant increase of ADAM12 in both SSc-MSC and -FB, with the higher levels observed in dcSSc cells. After ADAM12 silencing, the TGF- $\beta$ ability to upregulate $\alpha$-smooth muscle actin in both SSc-MSC and SSc-FB was inhibited. Conclusion. Our results suggest that in SSc, pericytes that transdifferentiate toward activated FB are present in the vascular tree, and TGF- $\beta$, while increasing ADAM12 expression, may modulate this transdifferentiation. (First Release June 1 2016; J Rheumatol 2016;43:1340-9; doi:10.3899/ jrheum.150996)
\end{abstract}

Key Indexing Terms:

SYSTEMIC SCLEROSIS

PERICYTE

activation and proliferation of resident fibroblasts (FB) associated with abnormal myofibroblast recruitment and proliferation, resulting in increased collagen deposition and leading to progressive organ dysfunction ${ }^{2,3,4,5}$.
From the Department of Applied Clinical Sciences and Biotechnology, Rheumatology Unit, School of Medicine, and the Department of Applied Clinical Sciences and Biotechnology, General Pathology Unit, University of L'Aquila, L'Aquila; Dipartimento Biomedico di Medicina Interna e Specialistica, Sezione di Reumatologia, Università degli Studi di Palermo, Palermo, Italy.

P. Cipriani, MD, PhD, Department of Applied Clinical Sciences and Biotechnology, Rheumatology Unit, School of Medicine, University of L'Aquila; P. Di Benedetto, PhD, Department of Applied Clinical Sciences and Biotechnology, Rheumatology Unit, School of Medicine, University of L'Aquila; P. Ruscitti, MD, Department of Applied Clinical Sciences and Biotechnology, Rheumatology Unit, School of Medicine, University of L'Aquila; V. Liakouli, MD, PhD, Department of Applied Clinical Sciences and Biotechnology, Rheumatology Unit, School of Medicine, University of L'Aquila; O. Berardicurti, MD, Department of Applied Clinical Sciences and Biotechnology, Rheumatology Unit, School of Medicine, University of L'Aquila; F. Carubbi, MD, Department of Applied Clinical Sciences and Biotechnology, Rheumatology Unit, School of Medicine, University of
L'Aquila; F. Ciccia, MD, PhD, Dipartimento Biomedico di Medicina Interna e Specialistica, Sezione di Reumatologia, Università degli Studi di Palermo; G. Guggino, MD, Dipartimento Biomedico di Medicina Interna e Specialistica, Sezione di Reumatologia, Università degli Studi di

Palermo; F. Zazzeroni, PhD, Department of Applied Clinical Sciences and Biotechnology, General Pathology Unit, University of L'Aquila; E. Alesse, MD, PhD, Department of Applied Clinical Sciences and Biotechnology, General Pathology Unit, University of L'Aquila; G. Triolo, MD, PhD, Dipartimento Biomedico di Medicina Interna e Specialistica, Sezione di Reumatologia, Università degli Studi di Palermo; R. Giacomelli, MD, PhD, Department of Applied Clinical Sciences and Biotechnology, Rheumatology Unit, School of Medicine, University of L'Aquila.

Address correspondence to Dr. P. Cipriani, Department of Applied Clinical Sciences and Biotechnology, Rheumatology Unit, School of Medicine, University of L'Aquila, Delta 6 Building, Via dell'Ospedale, 67100 L'Aquila, Italy.E-mail: paola.cipriani@cc.univaq.it

Accepted for publication April 8, 2016. 
During normal conditions, myofibroblasts, characterized by the presence of stress fibers containing $\alpha$-smooth muscle actin $(\alpha-S M A)$, are involved in extracellular matrix (ECM) deposition and wound contraction during wound healing ${ }^{6,7}$. Of note, when compared with normal skin, SSc skin shows a significant increase in myofibroblasts. The origin of activated myofibroblasts in the skin of patients with SSc is still a matter of debate ${ }^{8,9,10,11}$. There is evidence that activated myofibroblasts may arise from local conversion of dermal FB and it is also possible that resident and circulating mesenchymal progenitors may transdifferentiate toward activated myofibroblasts $^{12,13}$. Finally, we demonstrated that SSc-endothelial cells (SSc-EC), under the synergistic effect of transforming growth factor- $\beta$ (TGF- $\beta$ ) and endothelin 1 , may further transdifferentiate toward myofibroblasts ${ }^{14}$.

It has been suggested that pericytes and perivascular progenitor cells are involved in the process that, starting from chronic microvascular damage, evolves to fibrosis $15,16,17,18$. In fact, lineage tracing experiments, in an experimental model of kidney fibrosis ${ }^{19}$, confirmed that these cells are the main source of myofibroblasts, and the perivascular progenitor cells, with a profibrotic function, may be identified by the expression of 1 specific marker, the isoform 12 of ADAM $(\mathrm{ADAM} 12)^{20}$. It is well known that the main expression of ADAM12 may be observed during embryonic morphogenesis of skeletal muscles and visceral organs, and intriguingly this molecule may be newly expressed in several human fibrotic diseases $21,22,23,24,25,26$. Previously, we showed that because mesenchymal progenitors, which are generally considered an alternative source of functional pericytes ${ }^{27,28}$, exhibit the same phenotype and ability to differentiate of mature pericytes obtained from patients with SSc, they are involved in the generation of myofibroblasts in this disease ${ }^{3,4,5,14}$.

Translating the results obtained in experimental fibrosis, in our paper we assessed the expression of ADAM12 on the perivascular cells in the skin of patients with dcSSc and the ability of TGF- $\beta$, the pivotal profibrotic cytokine in SSc, to modulate this expression. The evidence of an increased ADAM12 expression on pericytes, positively modulated by TGF- $\beta$, supports the hypothesis that pericytes, which already transdifferentiate toward activated myofibroblasts, are present in the skin of patients with SSc and that these cells, mirroring other models of fibrosis, may contribute to the FB accumulation in the fibrotic skin of these patients.

\section{MATERIALS AND METHODS}

Patients, controls, and skin biopsies. Full-thickness biopsy samples, $2 \times 0.5$ $\mathrm{cm}$, isolated from excisional biopsy, were obtained from clinically involved skin of one-third of the distal forearm of 20 patients with dcSSc according to LeRoy, et $a l^{29}$. All patients fulfilled the 2013 American College of Rheumatology/European League Against Rheumatism classification criteria for $\mathrm{SSc}^{30}$. Skin with a modified Rodnan skin score ${ }^{31}$ of $\geq 1$ was considered clinically involved.

Of our patients, $50 \%$ were in a very early phase of SSc, considering that the term early, at present, refers to an undifferentiated connective tissue disease at higher risk to develop SSc, rather than a time at the beginning of the disease, as suggested by the pivotal study by Koenig, et $\mathrm{l}^{32}$. We further divided our patients into 2 subsets: patients fulfilling the classification criteria in $<1$ year from the onset of Raynaud phenomenon [early-onset subset (EOS), $\mathrm{n}=10$ ], and all the others [longstanding subset (LSS), $\mathrm{n}=$ 10]. Skin samples from the same region of 10 age- and sex-matched healthy controls (HC) who underwent surgical treatment for trauma were used as controls. The skin samples were processed for immunofluorescence (IF) and FB cell isolation and culture. All patients with SSc underwent a 20-day washout from any immunosuppressive treatment and 1 month from intravenous prostanoids before performing skin biopsy. During this period, only proton-pump inhibitors and clebopride were allowed. Patients who could not undergo therapeutic washout because of severe organ complications were not enrolled in our study. Biopsies were taken after informed consent, and the study was approved by our local committee. Demographic and clinical characteristics of the patients are shown in Table 1. For IF, the specimens were fixed in $10 \%$ buffered formalin, dehydrated in graded alcohol series, and embedded in paraffin.

Immunofluorescence. The IF analysis was performed on paraffin sections (3- $\mu \mathrm{m}$ thickness) using an anti-ADAM12 antibody (Novus). Antigen retrieval was carried out using Target Retrieval Solution (Dako). The immunoreaction was revealed using a secondary antibody (Alexa fluor 488, Sigma-Aldrich). Negative controls were obtained by omitting the primary antibody. Vasculature pericytes were highlighted using a Cy3 conjugated mouse monoclonal anti- $\alpha$-SMA antibody (Sigma-Aldrich) and an unconjugated anti-NG2 antibody (Santa Cruz, Biotechnology), EC using unconjugated anti-von Willebrand factor antibody (Dako), and FB using unconjugated anti-S100A4 antibody (Dako). The immunoreaction was revealed using a secondary antibody (Alexa fluor 555, Sigma-Aldrich). Cell nuclei were visualized using 4', 6-diamidino-2-phenylindole. Fluorescence was analyzed using an Olympus BX53 fluorescence microscope. The intensity of fluorescence was measured using ImageJ software [US National Institutes of Health (NIH)]. The median cell count was evaluated by counting the number of S100A4+/ADAM12+ cells in 5 different high power fields $(40 \times)$.

Isolation, culture, and immunophenotyping of MSC. After approval from the local ethics committee and written informed consent from patients, the bone marrow was obtained by aspiration from the posterior superior iliac crest from the patients enrolled in our study. Samples of MSC from bone marrow donors were used as controls.

MSC were obtained and expanded as previously described ${ }^{3}$. Third-passage MSC were analyzed for the surface expression of MSC antigens (CD45, CD73, CD90, CD34, CD79a, PDGFR- $\beta$ ) and pericyte markers ( $\alpha$-SMA, SM22 $\alpha$, NG2, desmin, RGS5), as previously described ${ }^{3}$ (data not shown).

FB isolation and culture. The skin specimen was placed into a 50-ml tube containing $10 \mathrm{ml}$ of collagenase (Sigma) at $37^{\circ} \mathrm{C}$ for $2 \mathrm{~h}$. After digestion, the samples were cultured in Dulbecco modified Eagle's medium (Sigma) supplemented with $10 \%$ fetal bovine serum (FBS; Standard South America origin), $100 \mathrm{units} / \mathrm{ml}$ penicillin, and $100 \mathrm{ng} / \mathrm{ml}$ streptomycin (Sigma) at $37^{\circ} \mathrm{C}$ in a humidified atmosphere of $5 \% \mathrm{CO}_{2}$. The isolated cells were analyzed for the surface expression of S100A4 antigen by flow cytometry (FACScan, Becton Dickinson) to assess their purity (S100A4+ cells $>99 \%$ ).

MSC and FB treatment with TGF- $\beta$. The optimal concentration of TGF- $\beta$ (R\&D) was established with a dose/response curve evaluating ADAM12 mRNA expression in both MSC and FB after TGF- $\beta$ administration for 7 days. The curve was performed using the MSC of $3 \mathrm{HC}$. Each experiment was performed in triplicate. The optimal stimulation dose for TGF- $\beta$ was 10 $\mathrm{ng} / \mathrm{ml}$ (data not shown). Both dcSSc and $\mathrm{HC}$ cells were cultured for 7 days in $1 \%$ FBS medium supplemented with optimal dose of TGF- $\beta$. Medium was changed every 2 days.

Small interfering RNA (siRNA) assay. dcSSc-MSC were seeded $24 \mathrm{~h}$ prior to transfection. At $70 \%$ confluence, the cells were transfected with ADAM12 siRNA (Life Technologies) or with Negative Control no-targeting scrambled

Personal non-commercial use only. The Journal of Rheumatology Copyright $\subset$ C 2016. All rights reserved. 
Table 1. Clinical and demographic features of the 20 patients with dcSSc. The internal organ involvement refers to the time of the biopsies.

\begin{tabular}{|c|c|c|c|c|c|c|}
\hline $\begin{array}{l}\text { Sex/Age, } \\
\text { Yrs }\end{array}$ & $\begin{array}{l}\text { Yr of SSc Onset/disease } \\
\text { Duration at Skin Biopsy }\end{array}$ & $\begin{array}{c}\mathrm{mRSS} / \mathrm{score} \\
\text { at Skin Biopsy }\end{array}$ & Autoantibodies & $\begin{array}{c}\text { Lung Involvment, } \\
\text { HRCT/PFT }\end{array}$ & $\begin{array}{c}\text { Heart Involvement/ } \\
\text { scleroderma Renal Crisis }\end{array}$ & $\begin{array}{c}\text { Raynaud } \\
\text { Phenomenon/ } \\
\text { digital Ulcers }\end{array}$ \\
\hline $\mathrm{F} / 22$ & $2013 / 1$ & $13 / 1$ & ANA/Scl-70 & Normal/normal & Normal/no & Yes/yes \\
\hline $\mathrm{F} / 31$ & $2014 / 1$ & $08 / 2$ & ANA/Scl-70 & Normal/normal & Normal/no & Yes/no \\
\hline $\mathrm{F} / 38$ & $2012 / 1$ & $09 / 2$ & ANA/Scl-70 & Normal/normal & $\mathrm{PAH} / \mathrm{no}$ & Yes/yes \\
\hline $\mathrm{F} / 31$ & $2013 / 1$ & $10 / 1$ & ANA/Scl-70 & Normal/normal & Normal/no & Yes/no \\
\hline $\mathrm{F} / 21$ & $2014 / 1$ & $09 / 1$ & ANA/Scl-70 & Normal/normal & Normal/no & Yes/no \\
\hline $\mathrm{F} / 31$ & $2012 / 1$ & $14 / 1$ & ANA/Scl-70 & Normal/normal & Normal/no & Yes/no \\
\hline $\mathrm{F} / 42$ & $2014 / 1$ & $16 / 2$ & ANA/Scl-70 & Fibrosis/normal & Normal/no & Yes/no \\
\hline $\mathrm{F} / 45$ & $2010 / 4$ & $17 / 2$ & ANA/Scl-70 & Normal/normal & Normal/no & Yes/no \\
\hline $\mathrm{F} / 21$ & $2009 / 5$ & $15 / 1$ & ANA/Scl-70 & Normal/normal & Normal/no & Yes/no \\
\hline $\mathrm{M} / 26$ & $2007 / 6$ & $10 / 2$ & ANA/Scl-70 & Fibrosis/normal & Normal/no & Yes/yes \\
\hline $\mathrm{F} / 21$ & $2009 / 4$ & $11 / 1$ & ANA/Scl-70 & Normal/normal & Normal/no & Yes/no \\
\hline $\mathrm{F} / 30$ & $2010 / 3$ & $12 / 2$ & ANA/Scl-70 & Fibrosis/normal & Normal/no & Yes/yes \\
\hline$F / 33$ & $2009 / 4$ & $12 / 1$ & ANA/Scl-70 & Normal/normal & Normal/no & Yes/no \\
\hline
\end{tabular}

SSc: systemic sclerosis; dcSSc: diffuse cutaneous SSc; mRSS: modified Rodnan skin score (maximum possible score = 51); HRCT: high-resolution computed tomography; PFT: pulmonary function test; ANA: antinuclear antibodies; Scl-70: topoisomerase; PAH: pulmonary arterial hypertension.

siRNA (scr; Life Technologies) using Lipofectamine 2000 reagent (Life Technologies). MSC were transfected for $24 \mathrm{~h}$ with $50 \mathrm{pmol}$ of siRNA in 2 $\mathrm{ml}$ of OptiMem. After incubation, cells were allowed to recover in normal growth conditions for $24 \mathrm{~h}$ post-transfection.

dcSSc-FB were transfected with the same siRNA construct used for MSC, and the transfection was performed using Lipofectamine 3000 (Life Technologies). Briefly, FB were plated $24 \mathrm{~h}$ prior to transfection. At $70 \%$ confluence, the cells were transfected for $24 \mathrm{~h}$ with 25 pmol of siRNA in 2 $\mathrm{ml}$ of OptiMem. After incubation, cells were allowed to recover in normal growth conditions for $24 \mathrm{~h}$ post-transfection. The expression of ADAM12 was determined by quantitative real-time PCR (qRT-PCR).

Western blot. MSC and FB, before and after TGF- $\beta$ treatment, were pelleted, washed twice with phosphate buffered saline, lysed in lysis buffer (1\% Triton X-100, 0.5\% NP-40, 50 mM Tris-Cl, pH 7.5, 150 mM NaCl, 1 mM EDTA, supplemented with $1 \mathrm{mM}$ phenylmethylsulfonyl fluoride, $1 \mathrm{mM} \mathrm{NaF}, 1 \mathrm{mM}$ $\mathrm{Na} 3 \mathrm{VO} 4,5 \mu \mathrm{g} / \mathrm{ml}$ aprotinin, $5 \mu \mathrm{g} / \mathrm{ml}$ leupeptin) for $30 \mathrm{~min}$ and cleared by centrifugation. The protein concentration was calculated by Bradford protein assay reagent (Bio-Rad). Fifty micrograms of proteins were separated by sodium dodecyl sulfate-polyacrylamide gel and transferred to nitrocellulose membranes. After $1 \mathrm{~h}$ blocking at room temperature in blocking buffer [5\% non-fat milk in Tris-buffered saline/1\% tween 20 (TBS/T)] and after washing 3 times for $5 \mathrm{~min}$ each in TBS/T, the membranes were incubated overnight at $4{ }^{\circ} \mathrm{C}$ with the primary antibodies $\alpha$-SMA (Abcam) and ADAM12 antibody (Novus) diluted in 5\% bovine serum albumin in TBS/T. Following 3 washes with TBS/T, horseradish peroxidase-conjugated secondary antibodies (Santa Cruz Biotechnology) diluted in blocking buffer were added for $30 \mathrm{~min}$ at room temperature and washed 3 times with TBS/T. The detection was performed by enhanced chemiluminescence detection reaction (Amersham Pharmacia Biotechnology). All the signals were quantified by normalizing to the tubulin signal (CP06 Anti- $\alpha$-Tubulin Mouse mAb-DM1A). Immunoreactive bands were quantified with densitometry using ImageJ software $(\mathrm{NIH})$.

qRT-PCR analysis. Total RNA was extracted from TGF- $\beta$-treated and -untreated MSC and FB using TRIzol (Sigma) and reverse transcribed into complementary DNA with the ThermoScript reverse transcription-PCR system (Invitrogen). The qRT-PCR was run in triplicate. ADAM12 and GAPDH gene expression were assessed by commercial Taqman gene expression assay (Hs01106101 and Hs02758991, respectively). CollA1, $\alpha-S M A$, and $C T G F$ gene expression was performed using SYBR green kits (Applied Biosystems). Primers were designed on the basis of the reported sequences [Primer bank from the National Center for Biotechnology Information: $\beta$-actin: 5'-CCT GGC ACC CAG CAC AAT-3' (forward) and 5'-AGT ACT CCG TGT GGA TCG GC-3' (reverse); $\alpha$-SMA: 5'-CGG TGC TGT CTC TCT ATG CC-3' (forward) and 5'-CGC TCA GTC AGG ATC TTC A-3' (reverse); Col1A1: 5'-AGG GCC AAG ACG AAG ACA GT-3' (forward) and 5'-AGA TCA CGT CAT CGC ACA ACA-3' (reverse); CTGF: 5'-CAG CAT GGA CGT TCG TCT G-3' (forward) and 5'-AAC CAC GGT TTG GTC CTT GG-3' (reverse)]. Results were analyzed after 45 cycles of amplification using the ABI 7500 Fast Real Time PCR System.

Statistical analysis. GraphPad Prism 5.0 software were used for statistical analyses. Results are expressed as median (range). Because of the nonparametric distribution of our data, the Mann-Whitney U test was used as appropriate for analyses. Statistical significance was expressed by a p value $<0.05$.

\section{RESULTS}

ADAM12 expression in skin dcSSc. Figure 1 shows that ADAM12 expression in dcSSc skin was significantly higher when compared with HC skin. Further, in LSS dcSSc skin, the fluorescence intensity of ADAM12 expression evaluated using ImageJ was higher than that observed in EOS (Figure 1: B1-6, C1-6, and D).

ADAM12 was strongly expressed in pericytes, EC, and FB of dcSSc skin. A weak positivity of this molecule was observed in the same cells of HC skin.

In dcSSc skin, the number of S100A4+/ADAM12+ FB surrounding the vessels was significantly higher than in HC 

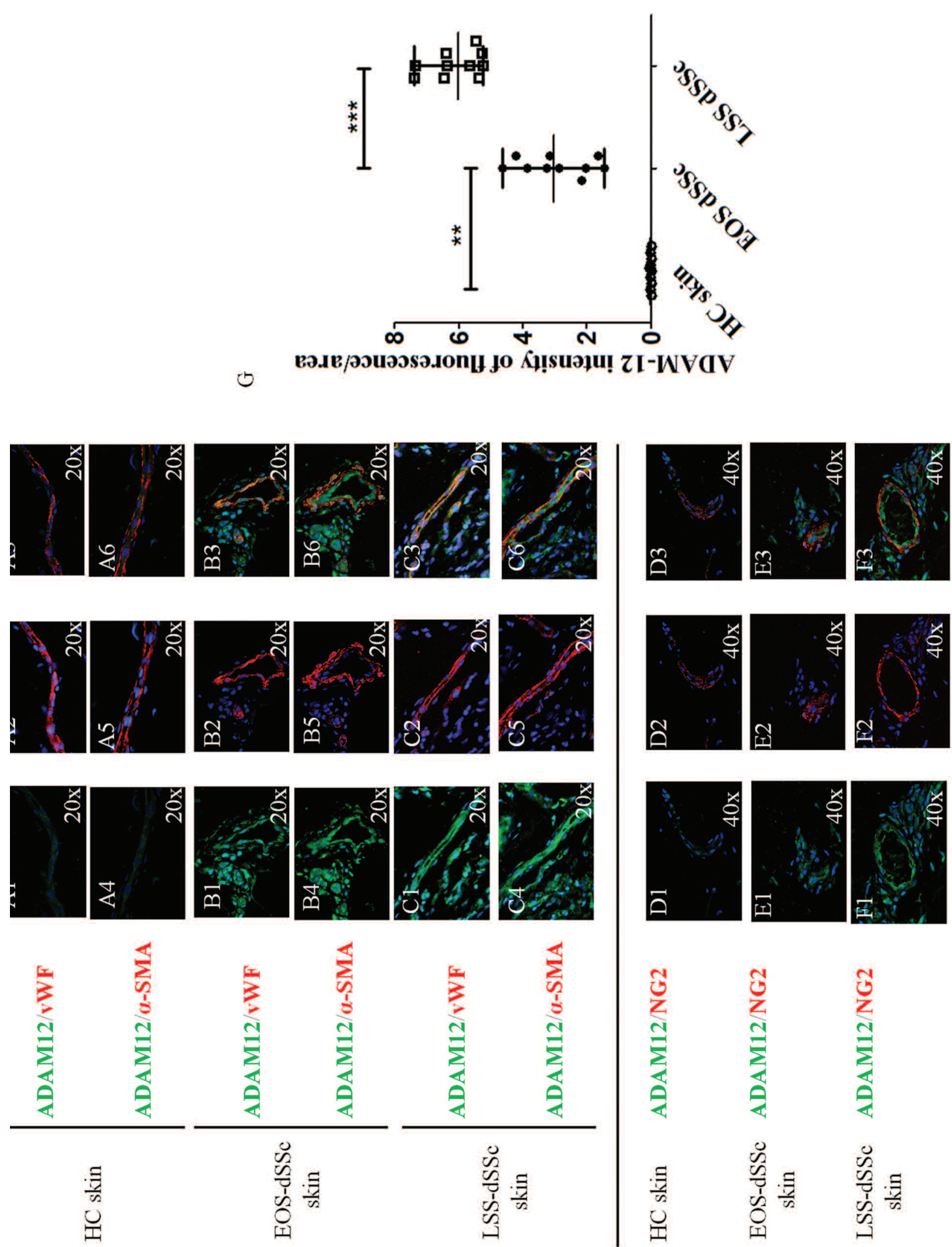

Personal non-commercial use only. The Journal of Rheumatology Copyright @ 2016. All rights reserved. 
Figure 1. ADAM12 expression in skin of patients with dcSSc. (A1-6) IF staining of HC skin. Microphotographs A1-3 show the same section stained with ADAM12 (green), vWF (red), and together. Microphotographs A4-6 show the same section stained with ADAM12 (green), $\alpha$-SMA (red), and together. A weak expression of ADAM12 may be observed in EC and pericytes of HC skin vessels. (B1-6) IF staining of EOS dcSSc skin. Microphotographs B1-3 show the same section stained with ADAM12 (green), vWF (red), and together. Microphotographs B4-6 show the same section stained with ADAM12 (green), $\alpha$-SMA (red), and together. ADAM12 was strongly expressed in EC and pericytes of EOS deSSc skin vessels. (C1-6) IF staining of LSS dcSSc skin. Microphotographs C1-3 show the same section stained with ADAM12 (green), vWF (red), and together. Microphotographs C4-6 show the same section stained with ADAM12 (green), $\alpha$-SMA (red), and together. ADAM12 was strongly expressed in EC and pericytes of LSS dcSSc skin vessels. (D1-3) IF staining of HC skin. Microphotographs show the same section stained with ADAM12 (green), NG2 (red), and together. A weak expression of ADAM12 may be observed in EC and pericytes of HC skin vessels. (E1-3) IF staining of EOS dcSSc skin. Microphotographs show the same section stained with ADAM12 (green), NG2 (red), and together. ADAM12 was strongly expressed in EC and pericytes of EOS dcSSc skin vessels. (F1-3) IF staining of LSS dcSSc skin. Microphotographs show the same section stained with ADAM12 (green), NG2 (red), and together. ADAM12 was strongly expressed in EC and pericytes of LSS dcSSc skin vessels. (G) Densitometric analysis of the IF intensity for ADAM12. Results are expressed as median (range) of the IF intensity measured using ImageJ. A significant increase of ADAM12 expression was observed in patients with dcSSc when compared with HC. HC vs EOS dcSSc: $* * p=0.0002$. HC vs LSS dcSSc: *** $\mathrm{p}=0.0001$. dcSSc: diffuse cutaneous systemic sclerosis; IF: immunofluorescence; vWF: von Willebrand factor; EC: endothelial cells; HC: healthy controls; EOS: early-onset subset; LSS: longstanding subset; $\alpha$-SMA: $\alpha$-smooth muscle actin.

skin (Figure 2). Further, in the LSS dcSSc skin, the number of S100A4+/ADAM12+ FB surrounding the vessels (Figure 2, C1-2) was significantly higher when compared with EOS dcSSc skin (Figure 2, B1-2).

TGF- $\beta$ induced an increased expression of ADAM12 in MSC and $F B$ isolated by patients with $d c S S c$. Figure 3 A shows that in untreated (UT) dcSSc-MSC, the mRNA levels of ADAM12 were significantly higher when compared with the levels of ADAM12 expression in UT HC-MSC [ADAM12 mRNA levels in UT MSC: $2.87(2.27-3.95)$ in EOS dcSSc-MSC vs $0.98(0.74-1.50)$ in HC-MSC, $\mathrm{p}<0.0001$, and 4.06 (2.65-4.34) in LSS dcSSc-MSC vs $0.98(0.74-1.50)$ in HC-MSC, $\mathrm{p}<0.0001]$. After $10 \mathrm{ng} / \mathrm{ml}$ of TGF- $\beta$ treatment for 7 days, we saw a significant increase in ADAM12, with the highest levels observed in dcSSc-MSC when compared with HC-MSC. [ADAM12 mRNA levels in TGF- $\beta$-treated MSC: 7.61 (6.23-9.56) in EOS dcSSc-MSC vs 3.42 (2.56-3.98) ADAM12 mRNA levels in HC-MSC, p < 0.0001 , and 9.65 (8.36-10.43) in LSS dcSSc-MSC vs 3.42 (2.56-3.98) ADAM12 mRNA levels in HC-MSC, p < 0.0001]. No significant differences were observed in ADAM12 expression between EOS and LSS dcSSc-MSC. The results obtained with perivascular MSC mirrored those observed in FB. The ADAM12 levels in UT HC-FB were
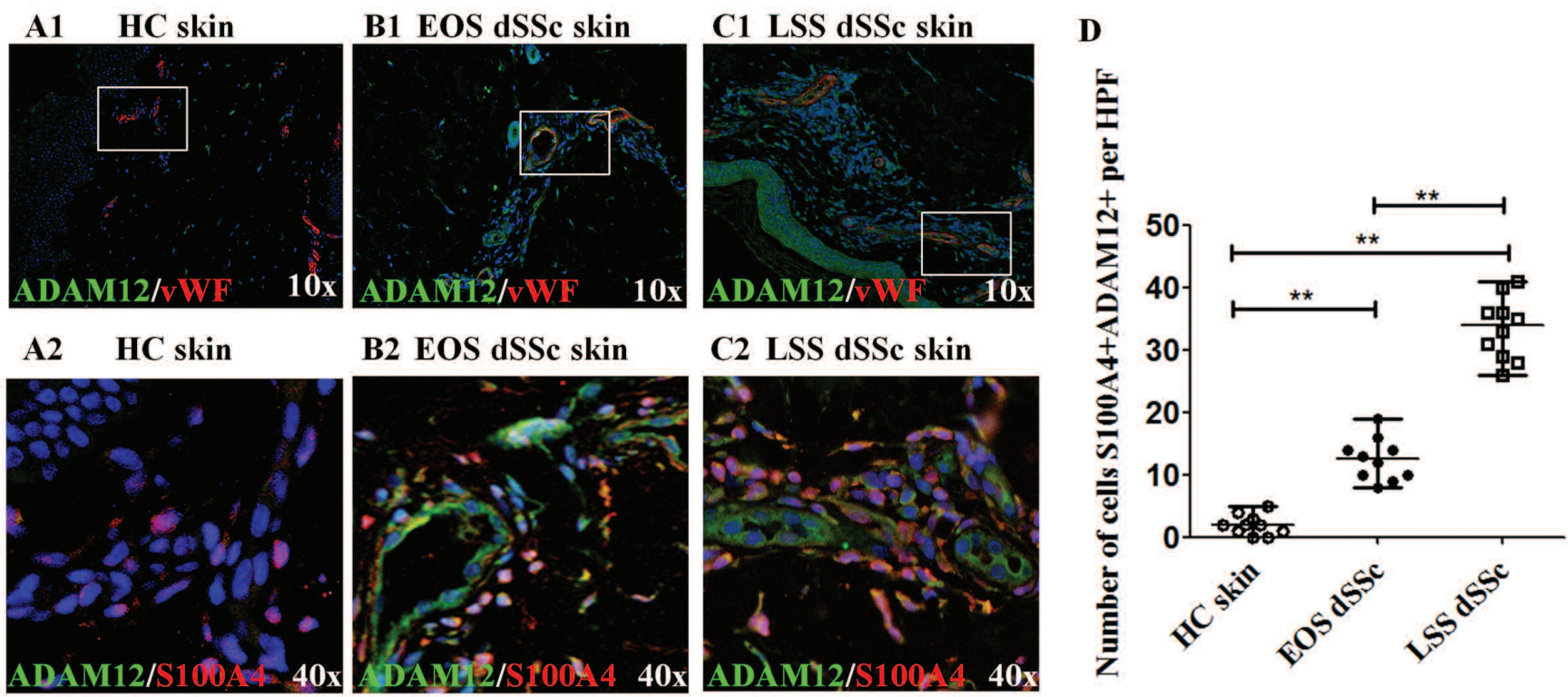
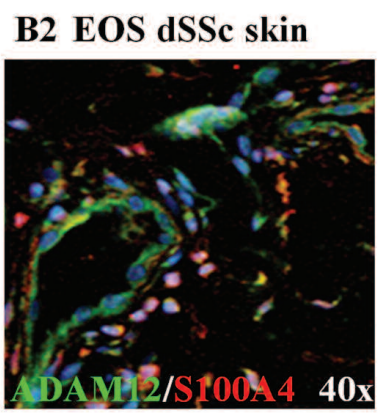

\section{C2 LSS dSSe skin}

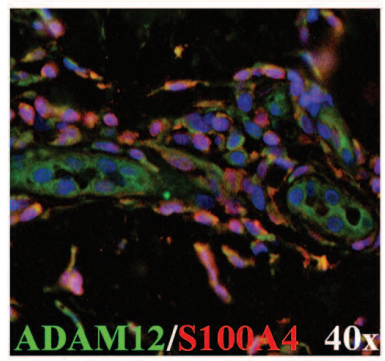

Figure 2. ADAM12 expression in perivascular FB of dcSSc skin. (A-C) IF staining of HC skin (A1-2), EOS dcSSc skin (B1-2), and LSS dcSSc skin (C1-2). The microphotographs A1, B1, and C1 show the merger of double-staining of ADAM12 (green) and vWF (red) at 10× magnification. The areas inside the squares are shown in microphotographs A2, B2, and C2 at 40× in the consecutive section and stained with ADAM12 (green) and S100A4 (red). The ADAM12+ perivascular cells coexpresses the FB marker S100A4. (D) Median number of S100A4+ADAM12+ cells obtained in 5 different HPF. The number of S100A4+ADAM12+ cells was significantly higher in LSS dcSSc skin when compared to EOS dcSSc skin. Any dot plot is representative of the median cells count per 5 HPF $(40 \times)$ for each patient. HC vs EOS dcSSc: $* *$ p $=0.0002$. HC vs LSS dcSSc: $* * p=0.0002$. EOS dcSSc vs LSS dcSSc: ** p $=0.0002$. FB: fibroblasts; dcSSc: diffuse cutaneous systemic sclerosis; IF: immunofluorescence; HC: healthy controls; EOS: early-onset subset; LSS: longstanding subset; vWF: von Willebrand factor; HPF: high power fields.

Personal non-commercial use only. The Journal of Rheumatology Copyright $@$ 2016. All rights reserved 

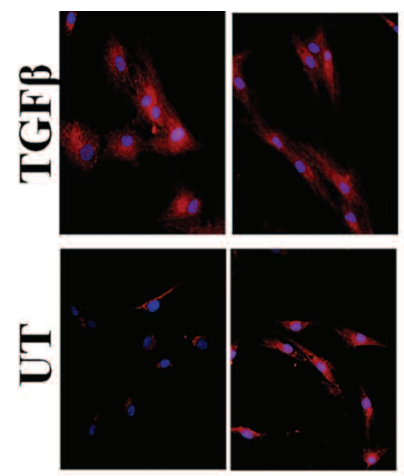

地

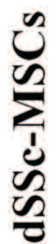

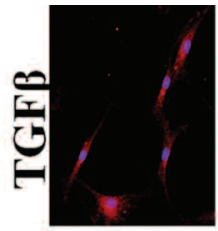
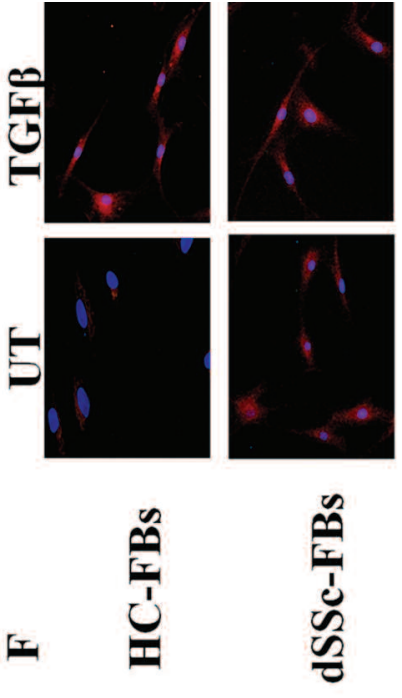

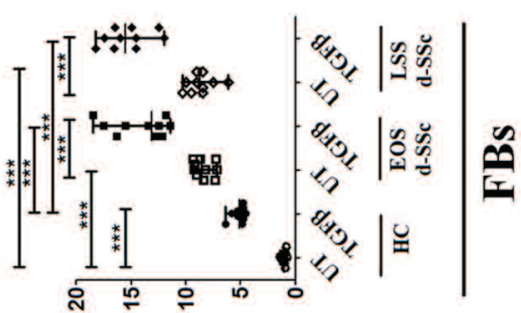

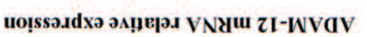

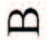

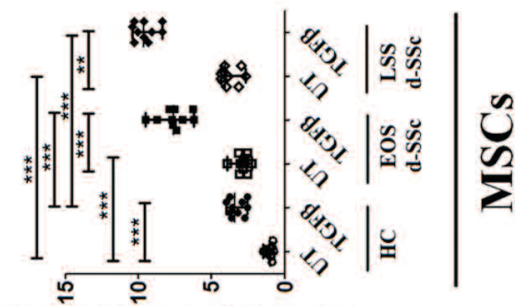

uo!ssว.ม

$\ll$

\section{(⿸丆口}

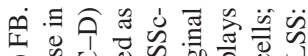

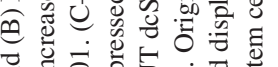

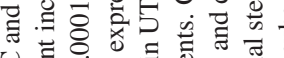

点

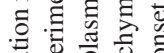
*

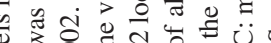

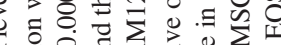

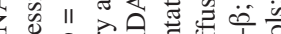
ص 10*

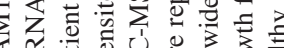

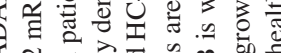

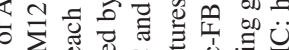

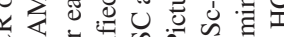

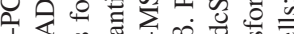
$\overrightarrow{\mathrm{\alpha}}$

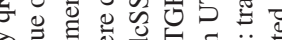

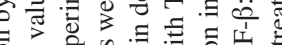

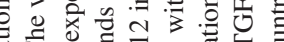

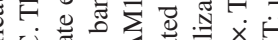
串

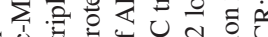
w

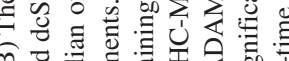

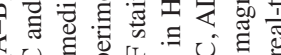
记

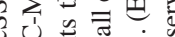
行

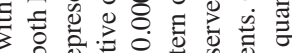

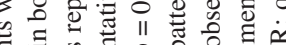

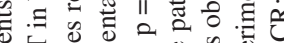

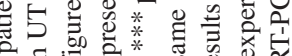

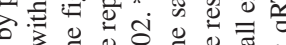

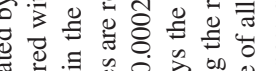

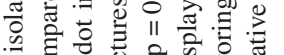

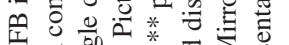
च छ

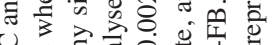

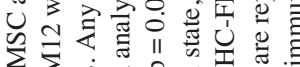

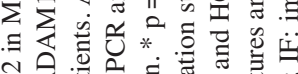

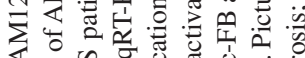

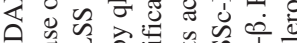

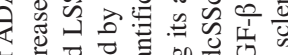

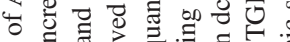
हี.

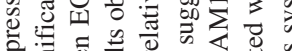

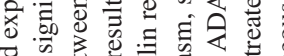
ठ

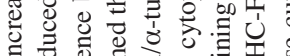

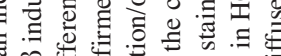

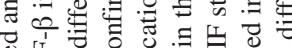

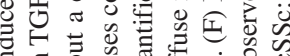

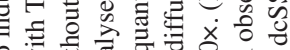

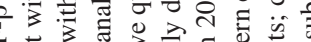

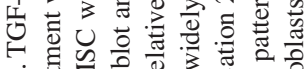

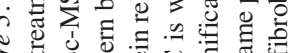

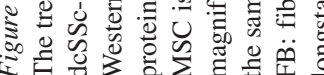


significantly lower than in dcSSc cells [ADAM12 mRNA levels in UT FB: 8.55 (7.19-9.33) in EOS dcSSc-FB vs 0.99 $(0.80-1.37)$ in HC-FB, $\mathrm{p}<0.0001$, and $8.73(6.12-10.30)$ in LSS dcSSc-FB vs $0.99(0.80-1.37)$ in HC-FB, p < 0.0001$]$. In HC-FB treated with TGF- $\beta$, we observed a significant increase of ADAM12 gene expression, and this increase was significantly higher in dcSSc-FB [ADAM12 mRNA levels in TGF- $\beta$-treated FB: 13.15 (11.43-18.45) in EOS dcSSc-FB vs 5.11 (4.56-6.36) ADAM12 mRNA levels in HC-FB, p < 0.0001 , and 15.50 (11.98-18.25) in LSS dcSSc-FB vs 5.11 (4.56-6.36) ADAM12 mRNA levels in HC-FB, p < 0.0001 ; Figure $3 \mathrm{~B}]$. These results were confirmed at the protein level by western blotting analyses (Figures $2 \mathrm{C}$ and 2D).

Immunolocalization of ADAM12 in dcSSc cells. Under normal conditions, inactive ADAM12 resides mainly in the perinuclear area, and it translocates to the cytoplasm when activated. This process may be induced by extracellular stimuli, such as TGF- $\beta^{33,34}$. In UT HC-MSC, the ADAM12 immunolocalization was in perinuclear regions. After activation with TGF- $\beta$ treatment, ADAM12 appeared evenly distributed in the cytoplasm of the HC-MSC. Interestingly, in UT dcSSc-MSC, ADAM12 was found on the cytoplasm, suggesting its activated status (Figure 3E). Mirroring the MSC behavior, UT dcSSc-FB displayed a cytoplasmic distribution of ADAM12. In UT HC-FB, ADAM12 was expressed in perivascular regions and TGF- $\beta$ treatment induced a cytoplasmic distribution of the molecule (Figure 3F). In both HC-MSC and HC-FB, the expression of ADAM12 was significantly lower when compared with dcSSc cells.

ADAM12 silencing inhibited TGF- $\beta$ induction of $\alpha$-SMA expression in SSc-MSC and -FB. To inactivate $A D A M 12$ gene product in both dcSSc-MSC and dcSSc-FB, we transfected the cells with ADAM12-siRNA or scrambled control siRNA (scr-siRNA). ADAM12-siRNA efficiently knocked down ADAM12 in both dcSSc-MSC and dcSSc-FB (> 70\%). Figures $4 \mathrm{~A}$ and $4 \mathrm{~B}$ show that TGF- $\beta$ stimulus induced a significant ADAM12 mRNA upregulation in both dcSSc-MSC and dcSSc-FB treated with scr-siRNA. But TGF- $\beta$ was unable to induce ADAM12 increase in cells treated with ADAM12-siRNA.

ADAM12 silencing blocked the ability of TGF- $\beta$ to induce $\alpha$-SMA protein expression in both dcSSc-MSC and dcSSc-FB, but in scr-siRNA-treated cells, TGF- $\beta$ treatment induced a significant upregulation of $\alpha$-SMA in both dcSSc-MSC and dcSSc-FB, as assessed by western blot (Figures 4C and 4D).

Further, in no-target, scr-siRNA-transfected dcSSc-FB, TGF- $\beta$ treatment induced a significant upregulation of CollAl, $\alpha$-SMA, and $C T G F$. However, ADAM12 silencing blocked the ability of TGF- $\beta$ to induce CollA1, $\alpha$-SMA, and CTGF gene expression in the same cells (Figures $4 \mathrm{E}, 4 \mathrm{~F}$, and 4G).

\section{DISCUSSION}

Our results show that pericytes of patients with SSc express the activated form of ADAM12 molecule, and that TGF- $\beta$, the main profibrotic cytokine in $\mathrm{SSc}^{26,35,36,37,38}$, modulates ADAM12 expression on these cells. These data suggest that, as observed in other experimental models of fibrosis, perivascular cells may be committed to transdifferentiate toward activated myofibroblasts and are involved in the fibrotic lesions of SSc.

After chronic injuries, fibrosis is the end stage in different pathologic conditions including cardiovascular diseases, chronic lung and kidney diseases, liver cirrhosis, and $\mathrm{SSc}^{39}$. To understand this complex biological process, studies ${ }^{12}$ suggested a role for the discrete and poorly appreciated populations of mesenchymal perivascular cells. These cells have been variously named as mural cells or pericytes, and several of their functions are largely unknown. At present, pericytes are considered one of the most important players in different fibrotic diseases ${ }^{4,40,41}$. Dulauroy, et $a l^{19}$, using genetic studies in mice to observe neural crest cell-derived embryonic mesenchyme, which expresses the ADAM12, found that fetal ADAM12+ cells contribute to the generation of perivascular cells in adult skeletal muscle. In fact, a subset of these cells deriving from the ADAM12+ lineage expressed pericytes markers and wrapped-around capillaries. After injury, the reactivation of ADAM12+ cells recapitulates an ontogenic program aimed at restoring vascular integrity. Under the influence of chronic stimuli, such as the persistence of profibrotic cytokines and/or chronic ischemia, this reparative mechanism may lead to an inappropriate fibrotic outcome characterized by the detachment and migration of ADAM12+ perivascular cells lineage to the tissue and their transdifferentiation toward activated myofibroblast. Of note, both the overexpression of profibrotic cytokines and the tissue ischemia are known to play pathogenic roles during SSc.

Our results confirm the presence of ADAM12+ cells in the perivascular areas of the affected skin of patients with dcSSc, and the expression of S100A4+/ADAM12+ cells in the fibrotic skin was limited to close to the vessels in EOS patients. Further, we showed an increased ADAM12 florescence intensity in LSS patients when compared with EOS patients, and this enhanced intensity was associated with an increased number of S100A4+/ADAM12+ cells and with the severity of the fibrosis. In addition, these cells in LSS patients were widely distributed in the fibrotic area.

In a mouse treated with bleomycin, an experimental model of SSc, it has been shown that pericytes may contribute to skin fibrosis ${ }^{16}$. Further, we provided evidence of a profibrotic phenotype of human perivascular cells expressing increased levels of $\alpha$-SMA and collagen during SSc ${ }^{3,5}$, and that SSc-EC may modulate the production of profibrotic molecules in perivascular dcSSc-MSC, thus eliciting a profibrotic phenotype ${ }^{4}$. The findings of ADAM12 hyperexpression and activation in SSc perivascular cells support the hypothesis of the profibrotic involvement of these cells during SSc.

Personal non-commercial use only. The Journal of Rheumatology Copyright @ 2016 . All rights reserved 

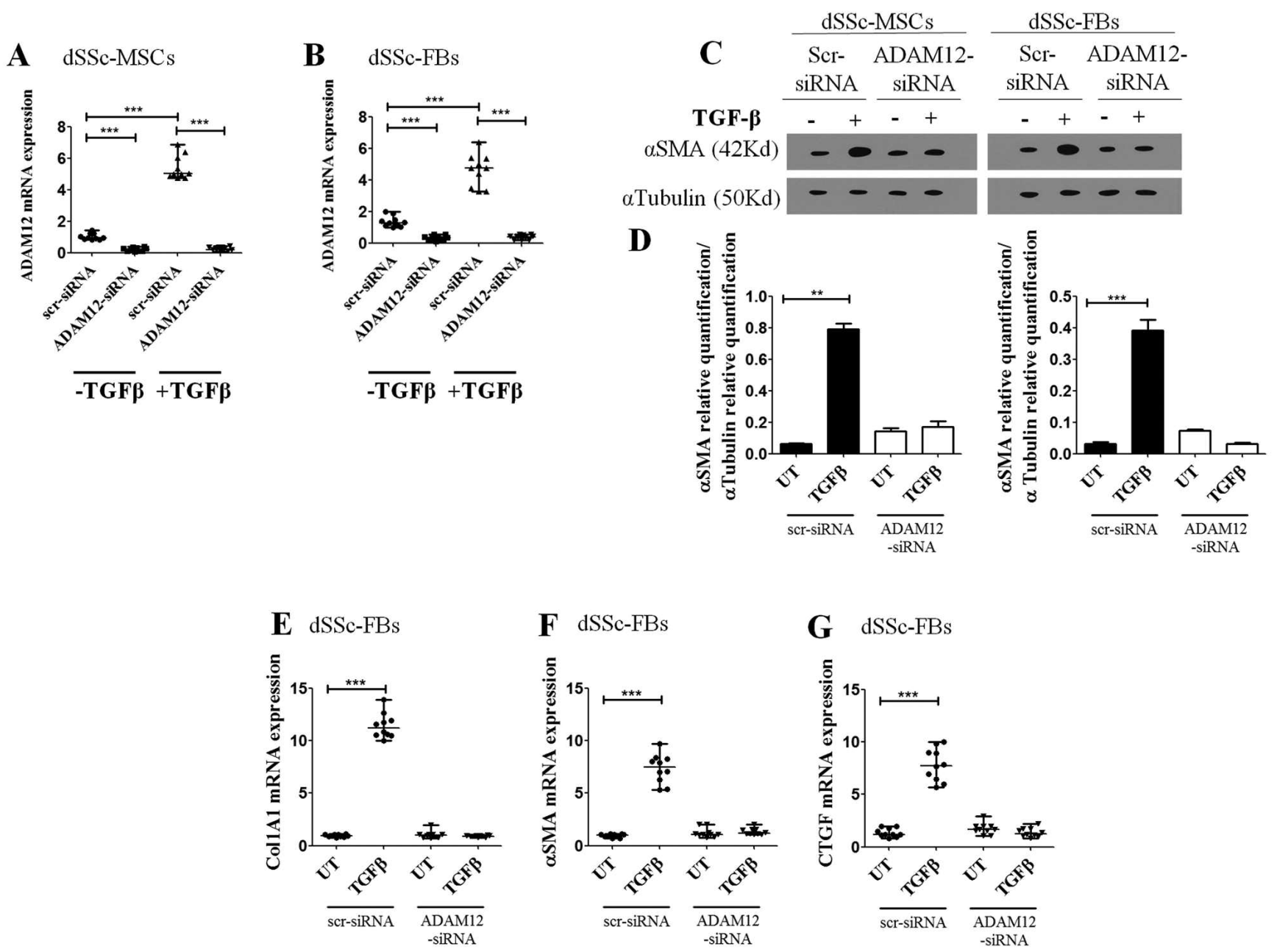

Figure 4. ADAM12 involvement in TGF- $\beta$-stimulated expression of myofibroblast markers. (A) dcSSc-MSC and (B) dcSSc-FB were transfected with specific ADAM12-siRNA or non-targeting scr, and ADAM12 expression was evaluated by qRT-PCR. The cells transfected with ADAM12-siRNA showed a decreased expression of ADAM12 gene when compared with cells transfected with scr-siRNA. The TGF- $\beta$ stimulus induced a significant increase of ADAM12 expression in both dcSSc-MSC and dcSSc-FB treated with scr-siRNA. On the contrary, in ADAM12-siRNA cells, TGF- $\beta$ was unable to induce ADAM12 increase. (C) Western blot of $\alpha$-SMA. In both dcSSc-MSC and dcSSc-FB treated with scr-siRNA, TGF- $\beta$ induced a significant increase of $\alpha$-SMA. On the contrary, after TGF- $\beta$ stimulation, ADAM12-siRNA transfected dcSSc-MSC and dcSSc-FB did not express $\alpha$-SMA protein. Pictures are representative of all experiments. (D) Densitometry analysis. Protein bands were quantified by densitometry and the values were expressed as protein relative quantification/ $\alpha$-tubulin relative quantification. $* * \mathrm{p}=0.0002$. $* * * \mathrm{p}=0.0001$. (E) mRNA expressions of CollA1, (F) $\alpha$-SMA, and (G) CTGF in dcSSc-FB, transfected with specific ADAM12-siRNA or non-targeting scr. dcSSc-FB transfected with ADAM12-siRNA did not show an increased expression of (E) CollA1, (F) $\alpha$-SMA, and (G) $C T G F$ after TGF- $\beta$ stimulation. On the contrary, in the cells treated with scr-siRNA, TGF- $\beta$ induced a significant increase of (E) CollAl, (F) $\alpha-S M A$, and (G) $C T G F$. Any single dot in the figures represents the median of triplicate experiments for each patient. TGF- $\beta$ : transforming growth factor- $\beta$; dcSSc: diffuse cutaneous systemic sclerosis; MSC: mesenchymal stem cells; FB: fibroblasts; siRNA: small interfering RNA; scr: scramble siRNA; qRT-PCR: quantitative real-time PCR; $\alpha$-SMA: $\alpha$-smooth muscle actin.

Taken together, these data lead us to speculate that perivascular cells of patients with dcSSc undergoing myofibroblast differentiation move from the perivascular areas to colonize the affected tissues and contribute to the FB recruitment and accumulation.

The functional involvement of ADAM12 in myofibroblast generation during SSc is still a matter of debate. ADAM12 plays a direct profibrotic role, regulating TGF- $\beta$ signaling $23,42,43$. After TGF- $\beta$ binds with specific receptors, the signal is transduced to the nucleus by members of the Sma- and Mad-related (Smad) family. It has been reported ${ }^{23,44}$ that, in vitro, TGF- $\beta$ stimulation induces an accumulation of ADAM12, in turn inducing Smad phosphorylation. On the other hand, depletion of endogenous ADAM12 by siRNA suppresses Smad phosphorylation ${ }^{44}$. In our setting, the levels of ADAM12 were significantly higher in SSc cells when compared with HC cells, already before the TGF- $\beta$ stimulation. After TGF- $\beta$ treatment, a significant increase of ADAM12 was shown in both SSc and HC cells, the highest levels observed in the cells of patients with SSc. We may suggest that, during SSc, a pathological environment enriched in TGF- $\beta$ may contribute to pericytes differentiation toward

Personal non-commercial use only. The Journal of Rheumatology Copyright @ 2016 . All rights reserved. 
myofibroblasts through ADAM12 upregulation, which acts as a positive regulator of profibrotic TGF- $\beta$ signaling.

The inactive form of ADAM12 is normally located in the perinuclear region of the cell. After TGF- $\beta$ stimulation, ADAM12 loses its prodomain, migrating into the cytoplasm as a mature molecule where it is catalytically active ${ }^{45}$. In our experiments, we showed that in both HC-MSC and HC-FB, the TGF- $\beta$ treatment induced an intracellular redistribution of ADAM12 from the perinuclear region to the cytoplasm. But in both dcSSc-MSC and dcSSc-FB, ADAM12 was already located in the cytoplasm, suggesting that during SSc, the pathological environment, enriched in TGF- $\beta$, may increase the active form of ADAM12.

In this activated status, ADAM12 may be involved in the transdifferentiation of both MSC and FB toward myofibroblasts, as shown by the increased $\alpha$-SMA expression. To confirm the functional role of ADAM12 in $\alpha$-SMA induction, after ADAM1 2 silencing and in vitro TGF- $\beta$ stimulation, we showed that the ADAM12-silenced cells were unable to induce $\alpha$-SMA expression. Further, after ADAM12 silencing, the SSc-FB were unresponsive to TGF- $\beta$ stimulation and were enabled to induce CollAl, $\alpha-S M A$, and CTGF gene expression.

Our results showed that not only pericytes, but also dcSSc-EC of the dermal vessel expressed ADAM12. The involvement of this molecule in EC is still not fully understood. During the physiological angiogenesis, many metalloproteinases are responsible for ECM degradation to support EC invasion ${ }^{46}$. Pathological activation of ADAM1 2 seems to be associated with the dissolution of adherent junctions and the loss of cell-cell contacts, resulting in EC apoptosis, as reported by an in vitro study performed on $\mathrm{EC}^{47,48}$. On these bases, we speculate that the increase of ADAM12 expression in a dysfunctional endothelium may be associated with the loss of cell-cell contacts, resulting in vessel rarefaction and avascular areas, as observed in SSc.

Several studies focused on pericytes as a possible source of the activated myofibroblasts observed during different fibrotic conditions, including SSc. In our work, we provide evidence of activated ADAM12 expression in SSc perivascular cells, suggesting their tendency toward profibrotic activity. Further, the evidence of S100A4+/ADAM12+ cells located in the perivascular areas during the early phase of the disease and widely diffused in the fibrotic areas during longstanding disease leads us to hypothesize their possible origin from perivascular cells that successively migrated into the affected tissues.

Further studies analyzing the relationship between ADAM12 and ALK5, or analyzing the inhibition of ADAM12 functions, may support the hypothesis that targeting ADAM12 can prevent fibrosis, a clinical pattern still needing effective therapies.

\section{ACKNOWLEDGMENT}

The authors thank Federica Sensini for her technical assistance.

\section{REFERENCES}

1. Denton CP, Black CM, Abraham DJ. Mechanisms and consequences of fibrosis in systemic sclerosis. Nat Clin Pract Rheumatol 2006;2:134-44.

2. Ho YY, Lagares D, Tager AM, Kapoor M. Fibrosis - a lethal component of systemic sclerosis. Nat Rev Rheumatol 2014; 10:390-402.

3. Cipriani P, Marrelli A, Benedetto PD, Liakouli V, Carubbi F, Ruscitti P, et al. Scleroderma mesenchymal stem cells display a different phenotype from healthy controls; implications for regenerative medicine. Angiogenesis 2013;16:595-607.

4. Cipriani P, Di Benedetto P, Ruscitti P, Campese AF, Liakouli V, Carubbi F, et al. Impaired endothelium-mesenchymal stem cells cross-talk in systemic sclerosis: a link between vascular and fibrotic features. Arthritis Res Ther 2014;16:442.

5. Cipriani P, Di Benedetto P, Capece D, Zazzeroni F, Liakouli V, Ruscitti P, et al. Impaired Cav-1 expression in SSc mesenchymal cells upregulates VEGF signaling: a link between vascular involvement and fibrosis. Fibrogenesis Tissue Repair 2014;7:13.

6. Tomasek JJ, Gabbiani G, Hinz B, Chaponnier C, Brown RA. Myofibroblasts and mechano-regulation of connective tissue remodelling. Nat Rev Mol Cell Biol 2002;3:349-63.

7. Ehrlich HP, Allison GM, Leggett M. The myofibroblast, cadherin, alpha smooth muscle actin and the collagen effect. Cell Biochem Funct 2006;24:63-70.

8. Wynn TA. Cellular and molecular mechanisms of fibrosis. J Pathol 2008;214:199-210.

9. Hinz B, Phan SH, Thannickal VJ, Galli A, Bochaton-Piallat ML, Gabbiani G. The myofibroblast: one function, multiple origins. Am J Pathol 2007;170:1807-16.

10. Ballhause TM, Soldati R, Mertens PR. Sources of myofibroblasts in kidney fibrosis: all answers are correct, however to different extent! Int Urol Nephrol 2014;46:659-64.

11. LeBleu VS, Taduri G, O'Connell J, Teng Y, Cooke VG, Woda C, et al. Origin and function of myofibroblasts in kidney fibrosis. Nat Med 2013;19:1047-53.

12. Duffield JS. The elusive source of myofibroblasts: problem solved? Nat Med 2012;18:1178-80.

13. Krieg T, Abraham D, Lafyatis R. Fibrosis in connective tissue disease: the role of the myofibroblast and fibroblast-epithelial cell interactions. Arthritis Res Ther 2007;9 Suppl 2:S4.

14. Cipriani P, Di Benedetto P, Ruscitti P, Capece D, Zazzeroni F, Liakouli $\mathrm{V}$, et al. The endothelial-mesenchymal transition in systemic sclerosis is induced by endothelin-1 and transforming growth factor- $\beta$ and may be blocked by macitentan, a dual endothelin-1 receptor antagonist. J Rheumatol 2015;42:1808-16.

15. Rajkumar VS, Sundberg C, Abraham DJ, Rubin K, Black CM. Activation of microvascular pericytes in autoimmune Raynaud's phenomenon and systemic sclerosis. Arthritis Rheum 1999; 42:930-41.

16. Liu S, Taghavi R, Leask A. Connective tissue growth factor is induced in bleomycin-induced skin scleroderma. J Cell Commun Signal 2010;4:25-30.

17. Shiwen X, Rajkumar V, Denton CP, Leask A, Abraham DJ. Pericytes display increased CCN2 expression upon culturing. J Cell Commun Signal 2009;3:61-4.

18. Ivarsson M, McWhirter A, Black CM, Rubin K. Impaired regulation of collagen pro-alpha 1(I) mRNA and change in pattern of collagen-binding integrins on scleroderma fibroblasts. J Invest Dermatol 1993;101:216-21.

19. Dulauroy S, Di Carlo SE, Langa F, Eberl G, Peduto L. Lineage tracing and genetic ablation of ADAM12(+) perivascular cells identify a major source of profibrotic cells during acute tissue injury. Nat Med 2012;18:1262-70.

20. Yagami-Hiromasa T, Sato T, Kurisaki T, Kamijo K, Nabeshima Y,

Personal non-commercial use only. The Journal of Rheumatology Copyright (c) 2016. All rights reserved 
Fujisawa-Sehara A. A metalloprotease-disintegrin participating in myoblast fusion. Nature 1995;377:652-6.

21. Gilpin BJ, Loechel F, Mattei MG, Engvall E, Albrechtsen R, Wewer UM. A novel, secreted form of human ADAM 12 (meltrin alpha) provokes myogenesis in vivo. J Biol Chem 1998;273:157-66.

22. Taniguchi T, Asano Y, Akamata K, Aozasa N, Noda S, Takahashi T, et al. Serum levels of ADAM12-S: possible association with the initiation and progression of dermal fibrosis and interstitial lung disease in patients with systemic sclerosis. J Eur Acad Dermatol Venereol 2013;27:747-53.

23. Le Pabic H, Bonnier D, Wewer UM, Coutand A, Musso O, Baffet G, et al. ADAM12 in human liver cancers: TGF-beta-regulated expression in stellate cells is associated with matrix remodeling. Hepatology 2003;37:1056-66.

24. Shi-Wen X, Renzoni EA, Kennedy L, Howat S, Chen Y, Pearson JD, et al. Endogenous endothelin-1 signaling contributes to type I collagen and $\mathrm{CCN} 2$ overexpression in fibrotic fibroblasts. Matrix Biol 2007;26:625-32.

25. Skubitz KM, Skubitz AP. Gene expression in aggressive fibromatosis. J Lab Clin Med 2004;143:89-98.

26. Lafyatis $\mathrm{R}$. Transforming growth factor $\beta$-at the centre of systemic sclerosis. Nat Rev Rheumatol 2014;10:706-19.

27. Cai X, Lin Y, Friedrich CC, Neville C, Pomerantseva I, Sundback $\mathrm{CA}$, et al. Bone marrow derived pluripotent cells are pericytes which contribute to vascularization. Stem Cell Rev 2009;5:437-45.

28. Bianco P, Riminucci M, Gronthos S, Robey PG. Bone marrow stromal stem cells: nature, biology, and potential applications. Stem Cells 2001;19:180-92.

29. LeRoy EC, Black C, Fleischmajer R, Jablonska S, Krieg T, Medsger TA Jr, et al. Scleroderma (systemic sclerosis): classification, subsets and pathogenesis. J Rheumatol 1988;15:202-5.

30. van den Hoogen F, Khanna D, Fransen J, Johnson SR, Baron M, Tyndall A, et al. 2013 Classification criteria for systemic sclerosis: an American College of Rheumatology/European League Against Rheumatism collaborative initiative. Ann Rheum Dis 2013; 72:1747-55.

31. Kahaleh MB, Sultany GL, Smith EA, Huffstutter JE, Loadholt CB, LeRoy EC. A modified scleroderma skin scoring method. Clin Exp Rheumatol 1986;4:367-9.

32. Koenig M, Joyal F, Fritzler MJ, Roussin A, Abrahamowicz M, Boire $\mathrm{G}$, et al. Autoantibodies and microvascular damage are independent predictive factors for the progression of Raynaud's phenomenon to systemic sclerosis: a twenty-year prospective study of 586 patients, with validation of proposed criteria for early systemic sclerosis. Arthritis Rheum 2008;58:3902-12.

33. Cao Y, Kang Q, Zhao Z, Zolkiewska A. Intracellular processing of metalloprotease disintegrin ADAM12. J Biol Chem 2002;277:26403-11.

34. Stautz D, Sanjay A, Hansen MT, Albrechtsen R, Wewer UM, Kveiborg M. ADAM12 localizes with c-Src to actin-rich structures at the cell periphery and regulates Src kinase activity. Exp Cell Res 2010;316:55-67.
35. Wan YN, Wang YJ, Yan JW, Li XP, Tao JH, Wang BX, et al. The effect of TGF- $\beta 1$ polymorphism on systemic sclerosis: a systematic review and pooled analysis of available literature. Rheumatol Int 2013;33:2859-65.

36. Hatton N, Frech T, Smith B, Sawitzke A, Scholand MB, Markewitz B. Transforming growth factor signalling: a common pathway in pulmonary arterial hypertension and systemic sclerosis. Int J Clin Pract Suppl 2011;172:35-43.

37. Liakouli V, Cipriani P, Marrelli A, Alvaro S, Ruscitti P, Giacomelli $\mathrm{R}$. Angiogenic cytokines and growth factors in systemic sclerosis. Autoimmun Rev 2011;10:590-4.

38. Rice LM, Padilla CM, McLaughlin SR, Mathes A, Ziemek J, Goummih S, et al. Fresolimumab treatment decreases biomarkers and improves clinical symptoms in systemic sclerosis patients. J Clin Invest 2015;125:2795-807.

39. Wynn TA. Common and unique mechanisms regulate fibrosis in various fibroproliferative diseases. J Clin Invest 2007;117:524-9.

40. Sato M, Suzuki S, Senoo H. Hepatic stellate cells: unique characteristics in cell biology and phenotype. Cell Struct Funct 2003;28:105-12.

41. Lin SL, Kisseleva T, Brenner DA, Duffield JS. Pericytes and perivascular fibroblasts are the primary source of collagen-producing cells in obstructive fibrosis of the kidney. Am J Pathol 2008;173:1617-27.

42. Ray A, Dhar S, Ray BK. Transforming growth factor-beta1-mediated activation of NF-kappaB contributes to enhanced ADAM-12 expression in mammary carcinoma cells. Mol Cancer Res 2010;8:1261-70.

43. Kim YM, Kim J, Heo SC, Shin SH, Do EK, Suh DS, et al. Proteomic identification of ADAM12 as a regulator for TGF- $\beta 1$-induced differentiation of human mesenchymal stem cells to smooth muscle cells. PLoS One 2012;7:e40820.

44. Atfi A, Dumont E, Colland F, Bonnier D, L'helgoualc'h A, Prunier $\mathrm{C}$, et al. The disintegrin and metalloproteinase ADAM12 contributes to TGF-beta signaling through interaction with the type II receptor. J Cell Biol 2007;178:201-8.

45. Loechel F, Gilpin BJ, Engvall E, Albrechtsen R, Wewer UM. Human ADAM 12 (meltrin alpha) is an active metalloprotease. J Biol Chem 1998;273:16993-7.

46. Roy R, Zhang B, Moses MA. Making the cut: protease-mediated regulation of angiogenesis. Exp Cell Res 2006;312:608-22.

47. Herren B, Levkau B, Raines EW, Ross R. Cleavage of beta-catenin and plakoglobin and shedding of VE-cadherin during endothelial apoptosis: evidence for a role for caspases and metalloproteinases. Mol Biol Cell 1998;9:1589-601.

48. Ponnuchamy B, Khalil RA. Role of ADAMs in endothelial cell permeability: cadherin shedding and leukocyte rolling. Circ Res 2008;102:1139-42. 